\title{
Estrategias para establecer un sistema que determine el seguimiento, estructura y evaluación de los trabajos final de grado en el Grado de Ingeniería en Diseño Industrial y Desarrollo de Productos de la UPV
}

E. Juliá Sanchis ${ }^{a}$, M. Valor Valor ${ }^{\text {, }}$ L. Pla Ferrando ${ }^{a}$, S. Sempere Ripoll ${ }^{\mathrm{a}}$, J.F. Picó Silvestre $^{\text {a }}$, M. Bonet-Aracila ${ }^{a}$, J.A. Martínez Cervera ${ }^{a}$, A. Jordá-Vilaplana ${ }^{a}$

${ }^{\text {a } U n i v e r s i t a t ~ P o l i t e ̀ c n i c a ~ d e ~ V a l e ̀ n c i a ~ 03801, ~ A l c o y, ~ S p a i n ~}$

erjusan@upv.es, mvalor@upv.es, mlpla@upv.es, ssempere@upv.es, juapisil@upv.es,

maboar@upv.es, jcerver@upv.es, amjorvi@upv.es

\begin{abstract}
The aim of this work is to establish a system that will determine the follow-up, structure and evaluation of final Degree Project Engineering in Industrial Design and Product Development, where a document has been prepared with the guidelines to be followed, Scheme with the recommendations according to the type of Final Degree Project (TFG) and the items to be taken into account by the professor to evaluate the TFG before its defense.
\end{abstract}

Keywords: final project, examination, signature, monitoring, structure, Industrial Design

\begin{abstract}
Resumen
El pretende trabajo trata de establecer un sistema que determine el seguimiento, la estructura y la evaluación de los trabajos final de grado en el Grado de Ingeniería en Diseño Industrial y desarrollo de productos, donde se han realizado un documento con las pautas a seguir, el esquema con las recomendaciones según el tipo de Trabajo fin de grado (TFG) y las rubricas a tener en cuenta por el profesor para evaluar el TFG antes de su defensa.
\end{abstract}

Palabras clave: Trabajo final de grado, evaluación, rubrica, seguimiento, estructura, diseño industrial.

\section{Introducción.}

La instauración de los nuevos planes de estudio ha supuesto un cambio en la manera de afrontar los Trabajos Final de Grado (TFG), estableciéndose, entre otros temas, nuevas competencias y capacidades, nuevos créditos asignados, nuevo sistema de funcionamiento y nueva normativa, lo cual preocupa a profesores y alumnos al no disponer de documentación que determine la nueva forma de proceder. 
En la actualidad no existe una sistematización del seguimiento, estructura y evaluación de los TFG en el Grado de Ingeniería en Diseño Industrial y Desarrollo de Productos en la Escuela Politécnica Superior de Alcoy, por lo que para mejorar el aprendizaje se considera necesario definir las estrategias que permitan establecer esos procedimientos.

Por otro lado, se ha detectado una dificultad derivada de la Normativa Marco de Trabajos Fin de Grado y Fin de Master de la UPV que obliga a que cada TFG tenga asignado un tutor encargado de orientar al alumno en el desarrollo del TFG (UPV, 2013). Esta exigencia hace que en la asignatura TFG se involucren muchos profesores que dirigen TFG de manera aislada y según el personal modo de entender la materia y que, como consecuencia, los alumnos recaban información de otros docentes con el fin de complementar la orientación del tutor y obtener TFG más completos e interdisciplinares. Estos docentes, que ayudan al alumno y complementan al tutor, actúan sin ningún tipo de reconocimiento docente y por lo tanto hay que buscar una solución a esta manera de proceder.

Al mismo tiempo, la manera de concebir el diseño industrial y el desarrollo de productos tiene ámbitos diversos que hacen necesario fijar las características de cada uno de ellos con tal de plantear posturas objetivas y comparables cara a la evaluación de los TFG.

Debido a toda esta problemática se plantea dentro de los Proyectos de Innovación y Mejora Educativa (PIME) del año 2016, promovidos y financiados por la Universidad Politécnica de Valencia (UPV), un proyecto mediante el cual se defina un sistema que especifique las estrategias a seguir para establecer el seguimiento, estructura, y evaluación de los TFG en los estudios de Ingeniería en Diseño Industrial y Desarrollo de Productos en la Escuela Politécnica Superior de Alcoy (EPSA).

\section{Objetivos.}

Con la finalidad de definir el alcance del proyecto se han establecido tres objetivos:

- Establecer un sistema que regule el procedimiento de tutoría y seguimiento de los TFG, definiendo los distintos actores y nivel de participación en el desarrollo de un TFG, así como las distintas fases que deben realizarse para un adecuado seguimiento de un TFG, indicando actores de cada una de ellas y duración estimada.

- Determinar la estructura de las distintas tipologías de TFG que pueden plantearse en el Grado en función del histórico disponible.

- Definir la metodología de evaluación de los TFG.

\section{Desarrollo de la Innovación.}

Para llevar a cabo el proyecto, dada la amplitud del mismo, se definen tres grupos de trabajo, cada uno de los cuales es el encargado de analizar la problemática de cada uno de los objetivos propuestos. Los grupos de trabajo se han denominado:

- Tutoría y seguimiento

(cc) EY-NC-ND 2017, Universitat Politècnica de València

Congreso IN-RED (2017) 
E. Juliá Sanchis, M. Valor Valor, L. Pla Ferrando, S. Sempere Ripoll, J.F. Picó Silvestre, M. BonetAracil, J.A. Martínez Cerver, A. Jordá Vilaplana

- Estructura

- Evaluación

Seguidamente se describe el trabajo desarrollado por cada uno de estos grupos.

\subsection{Tutoría y seguimiento.}

Este grupo tiene como tarea definir los distintos actores y su nivel de participación en el desarrollo de un TFG, al mismo tiempo que se precisan las distintas fases que deben realizarse para un adecuado seguimiento de un TFG, indicando actores de cada una de ellas y duración estimada. Son varias las propuestas de este grupo:

- Crear la modalidad de TFG coordinado por varios profesores que consiste en tutorizar los TFG en sesiones abiertas para los alumnos que opten por esta modalidad. Esta modalidad de tutorización no descarta el actual modelo de tutorización individual y pretende solucionar el problema que a menudo supone tutorizar un TFG un solo profesor.

- Preestablecer un horario para realizar las sesiones de tutoría de los TFG coordinados, de esta manera se pretende que el alumno vaya desarrollando el TFG desde el inicio del semestre.

- Establecer un seguimiento de las tutorías mediante una hoja de actividad, en donde se deben reflejar las indicaciones que los distintos profesores realizan sobre el trabajo que se les presenta a revisión.

\subsection{Estructura.}

Este otro grupo está encargado de determinar las distintas tipologías de TFG que pueden surgir en el Grado, así como concretar para cada tipo de TFG el contenido recomendable.

Para concretar las distintas tipologías se han analizado los 198 TFG presentados durante los cursos 2013/14, 2014/15 y 2015/16 distinguiéndose cuatro tipologías combinables de TFG:

- Diseño de producto

- Diseño interior

- Diseño textil

- Diseño de comunicación

Estas tipologías reflejan la demanda por parte del alumnado y del entorno socio-económico de la EPSA.

Es fundamental comprender que estas tipologías no son estancas, la experiencia evaluativa en anteriores TFG revela que, en la mayor parte de los proyectos defendidos, la materia se desarrolla de manera muy diversa y normalmente interrelacionando algunas de estas tipologías. Así por ejemplo un TFG de diseño textil puede contener un estudio con entidad propia relativo al diseño de un producto en donde se aplique el diseño textil, otro ejemplo podría ser el TFG de un producto en donde se desarrolla un estudio con entidad propia relativo al diseño de la comunicación del producto.

En cuanto a contenido y dadas las diferentes tipologías de TFG y con la finalidad de unificar la documentación a presentar se ha tomado en consideración lo indicado en la norma UNE 
Estrategias para establecer un sistema que determine el seguimiento, estructura y evaluación de los trabajos final de grado en el Grado de Ingeniería en Diseño Industrial y Desarrollo de Productos

157001:2014 Criterios generales para la elaboración formal de los documentos que constituyen un proyecto técnico lo indicado en la misma ha servido como punto de partida para definir los distintos documentos del TFG (AENOR, 2009). Recalcar que, dependiendo del tipo y alcance del proyecto, será o no necesario incluir la totalidad de los documentos ya que en última instancia será el tutor y la Comisión Académica del Título los que indique el contenido.

También se han considerado otros aspectos no contemplados en la norma con tal de enriquecer el contenido del TFG ya que se ha tenido en cuenta que los documentos del TFG constituyen un trabajo académico, algo diferente a un trabajo profesional ya que en este último basta describir la solución y en un trabajo académico hay que justificar y razonar la solución adoptada.

Como novedad, respecto a la documentación presentada hasta el momento en la EPSA en un TFG, se incluye un poster presentación con un resumen explicativo del proyecto. Este resumen del proyecto tiene por finalidad dar difusión a través de la UPV de los trabajos que desarrollan los alumnos mediante exposiciones, publicaciones impresas, divulgación por web, etc., sirviendo al mismo tiempo como curriculum para el alumno.

A modo de ejemplo se ofrece en (Tabla.1) los contenidos recomendados para un TFG de diseño de producto y en (Tabla. 2) los recomendados para el de comunicación.

Tabla. 1 Esquema contenidos recomendados de los TFG de DISEÑO DE PRODUCTOS.

\begin{tabular}{|c|c|}
\hline \multicolumn{2}{|l|}{ Portada normalizada } \\
\hline \multicolumn{2}{|c|}{ Resumen y palabras clave } \\
\hline Portada personalizada & Título, grado, alumno, curso, tutor/a \\
\hline \multicolumn{2}{|c|}{ Declaración de autoría y originalidad. } \\
\hline \multicolumn{2}{|c|}{$\begin{array}{l}\text { Documento de cesión de derechos y autorización para la difusión de trabajos } \\
\text { académicos }\end{array}$} \\
\hline Poster presentación & \\
\hline
\end{tabular}

\begin{tabular}{|c|c|c|c|}
\hline \multicolumn{4}{|c|}{ ÍNDICE GENERAL } \\
\hline \multirow[t]{6}{*}{ MEMORIA } & \multicolumn{3}{|c|}{1 Objeto y justificación } \\
\hline & \multicolumn{3}{|l|}{2 Antecedentes } \\
\hline & \multicolumn{3}{|c|}{3 Normas y referencias } \\
\hline & \multicolumn{3}{|c|}{4 Definiciones y abreviaturas } \\
\hline & \multirow{2}{*}{$\begin{array}{l}5 \text { Requisitos de } \\
\text { diseño }\end{array}$} & \multicolumn{2}{|c|}{ Descripción de las necesidades/p.c.i. } \\
\hline & & $\begin{array}{l}\text { Funciones del } \\
\text { producto }\end{array}$ & $\begin{array}{l}\text { Valoración de funciones / Valoración } \\
\text { entre funciones / Tablas de valoración de } \\
\text { funciones }\end{array}$ \\
\hline
\end{tabular}


E. Juliá Sanchis, M. Valor Valor, L. Pla Ferrando, S. Sempere Ripoll, J.F. Picó Silvestre, M. BonetAracil, J.A. Martínez Cerver, A. Jordá Vilaplana

\begin{tabular}{|c|c|c|c|c|}
\hline & \multicolumn{4}{|c|}{6 Análisis de soluciones } \\
\hline & \multirow{6}{*}{$\begin{array}{l}7 \text { Resultados } \\
\text { finales }\end{array}$} & \multicolumn{3}{|c|}{ Descripción y justificación del diseño adoptado } \\
\hline & & Viabilidad & \multicolumn{2}{|c|}{$\begin{array}{l}\text { Viabilidad técnica y física / Viabilidad } \\
\text { económica / Viabilidad financiera }\end{array}$} \\
\hline & & \multicolumn{3}{|c|}{ Diagrama sistémico del producto } \\
\hline & & \multicolumn{3}{|c|}{ Esquema de desmontaje del producto } \\
\hline & & \multicolumn{3}{|c|}{ Análisis estructural } \\
\hline & & \multicolumn{3}{|c|}{ Dimensionado previo } \\
\hline & \multicolumn{4}{|l|}{8 Conclusiones } \\
\hline \multirow[t]{7}{*}{ ANEXOS } & \multirow[t]{5}{*}{1 Prototipado } & Elementos & \multicolumn{2}{|c|}{$\begin{array}{l}\text { Elementos normalizados / Elementos } \\
\text { comerciales / Productos intermedios o } \\
\text { semielaborados / Elementos ya fabricados } \\
\text { por la empresa }\end{array}$} \\
\hline & & $\begin{array}{l}\text { Maquinas, } \\
\text { herramientas y } \\
\text { útiles }\end{array}$ & \multicolumn{2}{|c|}{ Para fabricación / Para el ensamblaje } \\
\hline & & \multicolumn{3}{|c|}{ Construcción de los elementos } \\
\hline & & \multicolumn{3}{|c|}{ Ensamblaje de subconjuntos } \\
\hline & & \multicolumn{3}{|c|}{ Acabado superficial } \\
\hline & \multicolumn{4}{|l|}{2 Cálculos } \\
\hline & \multicolumn{4}{|c|}{3 Otros documentos } \\
\hline \multirow[t]{3}{*}{ PLANOS } & $\begin{array}{l}1 \text { Planos de } \\
\text { conjunto. }\end{array}$ & \multicolumn{3}{|c|}{$\begin{array}{l}\text { Planos de conjunto, en proyección ortogonal, con marcas de } \\
\text { elementos, cotas generales y listado de elementos }\end{array}$} \\
\hline & $\begin{array}{l}2 \text { Planos de } \\
\text { subconjuntos }\end{array}$ & \multicolumn{3}{|c|}{$\begin{array}{l}\text { Planos de todos los subconjuntos con acotación funcional/ } \\
\text { para construcción }\end{array}$} \\
\hline & $\begin{array}{l}3 \text { Planos de } \\
\text { despiece }\end{array}$ & \multicolumn{3}{|c|}{$\begin{array}{l}\text { Planos de todos los elementos componentes con acotación } \\
\text { funcional / para construcción }\end{array}$} \\
\hline \multirow{2}{*}{\multicolumn{2}{|c|}{$\begin{array}{l}\text { PROTOTIPOS, MAQUETAS } \\
\text { Y/O MODELOS }\end{array}$}} & \multicolumn{2}{|c|}{ Presentación del producto } & $\begin{array}{l}\text { Perspectivas, simulaciones, } \\
\text { animaciones o similares }\end{array}$ \\
\hline & & \multicolumn{3}{|l|}{ Prototipo } \\
\hline \multicolumn{5}{|c|}{ PLIEGO DE CONDICIONES } \\
\hline \multicolumn{5}{|c|}{ ESTADO DE MEDICIONES/PRESUPUESTO } \\
\hline \multicolumn{5}{|c|}{ ESTUDIOS COMPLEMENTARIOS } \\
\hline
\end{tabular}

(c)) EY-NC-ND 2017, Universitat Politècnica de València 
Estrategias para establecer un sistema que determine el seguimiento, estructura y evaluación de los trabajos final de grado en el Grado de Ingeniería en Diseño Industrial y Desarrollo de Productos

Tabla 2 Esquema contenidos recomendados de los TFG de DISEÑO DE COMUNICACIÓN.

\begin{tabular}{|c|c|}
\hline \multicolumn{2}{|l|}{ Portada normalizada } \\
\hline \multicolumn{2}{|c|}{ Resumen y palabras clave } \\
\hline Portada personalizada & Título, grado, alumno, curso, tutor/a \\
\hline \multicolumn{2}{|c|}{ Declaración de autoría y originalidad. } \\
\hline \multicolumn{2}{|c|}{ Documento de cesión de derechos y autorización para la difusión de trabajos académicos } \\
\hline
\end{tabular}

\begin{tabular}{|c|c|c|c|c|c|}
\hline \multicolumn{6}{|c|}{ ÍNDICE GENERAL } \\
\hline \multirow[t]{18}{*}{ MEMORIA } & \multicolumn{5}{|c|}{1 Objeto y justificación. } \\
\hline & 2 & \multicolumn{2}{|c|}{ Antecedentes } & \multicolumn{2}{|c|}{$\begin{array}{l}\text { Gráficos, estilísticos y análisis de la } \\
\text { competencias }\end{array}$} \\
\hline & \multicolumn{5}{|c|}{3 Normas y referencias } \\
\hline & \multicolumn{5}{|c|}{4 Definiciones y abreviaturas } \\
\hline & 5 & \multicolumn{2}{|c|}{ Requisitos de diseño: } & \multicolumn{2}{|c|}{ Informe de instrucciones o Briefing: } \\
\hline & 6 & \multicolumn{4}{|c|}{$\begin{array}{l}\text { Análisis de soluciones: Documentación gráfica del proceso de ideación de } \\
\text { la comunicación }\end{array}$} \\
\hline & \multirow{12}{*}{\multicolumn{2}{|c|}{$\begin{array}{l}7 \text { Resultados } \\
\text { finales }\end{array}$}} & \multicolumn{3}{|c|}{ Descripción y justificación del diseño adoptado } \\
\hline & & & Aspectos & \multicolumn{2}{|c|}{ Herramientas y técnicas empleadas } \\
\hline & & & tecnicos & \multicolumn{2}{|c|}{ Aspectos técnicos de la imagen } \\
\hline & & & & \multirow[t]{3}{*}{ Arte final } & Soporte de la comunicación \\
\hline & & & & & $\begin{array}{l}\text { Características técnicas para su } \\
\text { reproducción }\end{array}$ \\
\hline & & & & & Procesos de impresión \\
\hline & & & Aspectos & \multicolumn{2}{|c|}{ Imagen: creación y tratamiento } \\
\hline & & & esteticos & \multicolumn{2}{|c|}{$\begin{array}{l}\text { Gráficos( logotipo, isotipo...): creación y } \\
\text { tratamiento }\end{array}$} \\
\hline & & & & \multicolumn{2}{|c|}{ Tipografía: selección, creación y tratamiento } \\
\hline & & & & \multicolumn{2}{|c|}{ Pruebas y variaciones cromáticas } \\
\hline & & & & \multicolumn{2}{|c|}{$\begin{array}{l}\text { Composición: pruebas de composición y/o } \\
\text { retículas }\end{array}$} \\
\hline & & & & \multicolumn{2}{|c|}{$\begin{array}{l}\text { Significado de la comunicación: adecuación de } \\
\text { la estética a las exigencias y/o eficacia } \\
\text { perseguida con el diseño de la comunicación. }\end{array}$} \\
\hline
\end{tabular}


E. Juliá Sanchis, M. Valor Valor, L. Pla Ferrando, S. Sempere Ripoll, J.F. Picó Silvestre, M. BonetAracil, J.A. Martínez Cerver, A. Jordá Vilaplana

\begin{tabular}{|l|l|l|}
\hline \multicolumn{2}{|l|}{8 Conclusiones } \\
\hline ANEXOS \\
\hline PLANOS \\
\hline $\begin{array}{l}\text { PROTOTIPOS, } \\
\text { MAQUETAS } \\
\text { Y/O } \\
\text { MODELOS }\end{array}$ & $\begin{array}{l}\text { Presentación } \\
\text { del producto }\end{array}$ & $\begin{array}{l}\text { Maquetas digitales y/o pruebas impresas (cartelería } \\
\text { dípticos, etiquetas...) sobre papel o volúmenes (envases, } \\
\text { fajitas retráctiles..) }\end{array}$ \\
\cline { 2 - 3 } & Prototipos \\
\hline PLIEGO DE CONDICIONES \\
\hline ESTADO DE MEDICIONES/ PRESUPUESTO \\
\hline ESTUDIOS COMPLEMENTARIOS \\
\hline
\end{tabular}

\subsection{Evaluación}

Este último grupo tiene por tarea definir la metodología de evaluación, debiendo establecer los criterios e instrumentos de evaluación del TFG. Para ello se han analizado diversas experiencias previas sobre metodologías de evaluación (CIESE, 2016), (Universidad de Granada ,2015), (UPC, 2013), (UPC, 2014) y artículos publicados en revistas educativas basadas en el TFG de distintas Universidades Nacionales,(ROISS, S. 2015) (Capó, A.J.; Guerrero, C.; Miró, J; Egea, A. 2012) (Valderrama, E.; Rullán, M.; Sánchez, F.; Pons, J.; Cores, F.; Bisbal, J. 2009), (Rekalde Rodríguez, I. 2011), (Bonilla Priego M.J.; Fuentes Moraleda, L.; Vacas Guerrero, C.; Vacas Guerrero, T.2012)

A partir de estas experiencias previas se han considerado fundamental evaluar tres aspectos del TFG: el desarrollo del trabajo, la documentación final y la defensa del proyecto. Cada uno de estos aspectos es objeto de evaluación mediante el empleo de rúbricas, ya que se ha considerado que este instrumento de evaluación permite al alumno conocer de antemano los detalles de cómo va a ser evaluado, además de ser objetivas y justificar mejor una calificación.

Para evaluar el desarrollo del trabajo se ha considerado que debe ser el tutor el que la lleva a cabo, de manera que evalúe el seguimiento, las capacidades y la documentación lograda. Por una parte, el tutor evalúa las competencias transversales empleando la rúbrica relativa a las Competencias Transversales que la UPV ha introducido en la aplicación EBRON (EBRON, 2014). Por otra parte, el tutor emite un informe del TFG también a través de la aplicación Ebrón. Este informe no forma parte de la calificación de la asignatura ya que su finalidad es servir de información al tribunal.

Otro de los aspectos a evaluar es la documentación presentada, en este caso es el tribunal asignado el que lleve a cabo la evaluación. La calificación de esta fase se realizará previamente a la defensa mediante la rúbrica (Tabla.3) y será la media aritmética de las calificaciones de cada miembro del tribunal. Tendrá un peso del $70 \%$ sobre la nota final. 
Estrategias para establecer un sistema que determine el seguimiento, estructura y evaluación de los trabajos final de grado en el Grado de Ingeniería en Diseño Industrial y Desarrollo de Productos

Tabla. 3 Rubrica para la evaluación de la documentación.

\begin{tabular}{|c|c|c|c|c|c|}
\hline & 1 INSUFICIENTE & $\begin{array}{c}2 \\
\text { REGULAR }\end{array}$ & $\begin{array}{c}3 \\
\text { BIEN }\end{array}$ & $\begin{array}{c}4 \\
\text { EXCELENTE }\end{array}$ & $\%$ \\
\hline $\begin{array}{l}\text { Organización } \\
\text { de la } \\
\text { memoria }\end{array}$ & $\begin{array}{l}\text { La información } \\
\text { está mal } \\
\text { organizada, no } \\
\text { existe una } \\
\text { continuidad lógica } \\
\text { en el contenido. }\end{array}$ & $\begin{array}{l}\text { En general la } \\
\text { información } \\
\text { apenas se } \\
\text { organiza de } \\
\text { manera lógica } \\
\text { encontrándose } \\
\text { varios párrafos, } \\
\text { subtítulos o títulos } \\
\text { relevantes } \\
\text { erróneamente } \\
\text { dispuestos. }\end{array}$ & $\begin{array}{l}\text { En general la } \\
\text { información está } \\
\text { bien organizada } \\
\text { aunque con algún } \\
\text { párrafo, subtitulo } \\
\text { o título relevante } \\
\text { erróneamente } \\
\text { dispuesto. }\end{array}$ & $\begin{array}{l}\text { La información } \\
\text { está bien } \\
\text { organizada con } \\
\text { párrafos bien } \\
\text { redactados y con } \\
\text { subtítulos, } \\
\text { siguiendo un índice } \\
\text { lógico. }\end{array}$ & $1 \%$ \\
\hline $\begin{array}{l}\text { Redacción, } \\
\text { expresión y } \\
\text { ortografía. }\end{array}$ & $\begin{array}{l}\text { El trabajo escrito } \\
\text { presenta muchos } \\
\text { errores } \\
\text { ortográficos, } \\
\text { gramaticales y de } \\
\text { expresión. }\end{array}$ & $\begin{array}{l}\text { El trabajo escrito } \\
\text { presenta unos } \\
\text { cuantos errores } \\
\text { ortográficos, } \\
\text { gramaticales y de } \\
\text { expresión. }\end{array}$ & $\begin{array}{l}\text { El trabajo escrito } \\
\text { presenta algún } \\
\text { error ortográfico, } \\
\text { gramatical y/o de } \\
\text { expresión muy } \\
\text { puntual. }\end{array}$ & $\begin{array}{l}\text { Está redactado de } \\
\text { forma clara y sin } \\
\text { errores } \\
\text { ortográfico. }\end{array}$ & $2 \%$ \\
\hline $\begin{array}{l}\text { Fuentes de } \\
\text { información }\end{array}$ & $\begin{array}{l}\text { Solo maneja } \\
\text { información } \\
\text { procedente de } \\
\text { internet que no es } \\
\text { fiable ni apropiada. } \\
\text { No usa un sistema } \\
\text { de citación y no la } \\
\text { integra en el trabajo. }\end{array}$ & $\begin{array}{l}\text { La bibliografía } \\
\text { es muy básica. } \\
\text { No usa un sistema } \\
\text { de citación y no } \\
\text { siempre se integra } \\
\text { adecuadamente en } \\
\text { el trabajo. }\end{array}$ & $\begin{array}{l}\text { La bibliografía } \\
\text { es de interés } \\
\text { para el trabajo, } \\
\text { pero incompleta. } \\
\text { El trabajo } \\
\text { presenta algunos } \\
\text { fallos en el } \\
\text { sistema de } \\
\text { citación o en la } \\
\text { integración de las } \\
\text { referencias } \\
\text { bibliográficas en } \\
\text { el trabajo escrito. }\end{array}$ & $\begin{array}{l}\text { La bibliografía es } \\
\text { la esencial e } \\
\text { importante. Usa } \\
\text { las referencias } \\
\text { bibliográficas de } \\
\text { forma correcta y en } \\
\text { el momento } \\
\text { apropiado durante } \\
\text { el trabajo escrito. }\end{array}$ & $2 \%$ \\
\hline $\begin{array}{l}\text { Objetivos del } \\
\text { trabajo }\end{array}$ & $\begin{array}{l}\text { Los objetivos son } \\
\text { confusos y/o no } \\
\text { adecuados al tema } \\
\text { y/o sin interés para } \\
\text { el grado. }\end{array}$ & $\begin{array}{l}\text { Varios objetivos } \\
\text { son imprecisos o } \\
\text { no adecuados al } \\
\text { tema y/o no se ha } \\
\text { justificado el } \\
\text { cumplimiento de } \\
\text { algún objetivo } \\
\text { planteado. }\end{array}$ & $\begin{array}{l}\text { Aunque los } \\
\text { objetivos son } \\
\text { claros y realistas } \\
\text { resultan de escaso } \\
\text { interés en el } \\
\text { grado. Se han } \\
\text { cumplido todos } \\
\text { los objetivos } \\
\text { planteados. }\end{array}$ & $\begin{array}{l}\text { Los objetivos son } \\
\text { claros, realistas, } \\
\text { adecuados al tema } \\
\text { y de interés } \\
\text { relevante en el } \\
\text { grado. Se han } \\
\text { cumplido todos los } \\
\text { objetivos } \\
\text { planteados. }\end{array}$ & $\begin{array}{l}15 \\
\%\end{array}$ \\
\hline $\begin{array}{l}\text { Elaboración } \\
\text { de } \\
\text { conclusiones }\end{array}$ & $\begin{array}{l}\text { Las conclusiones no } \\
\text { se vinculan con la } \\
\text { información que se } \\
\text { maneja y/o con la } \\
\text { realidad en que se } \\
\text { deben aplicar y/o no } \\
\text { son significativos. }\end{array}$ & $\begin{array}{l}\text { Las conclusiones } \\
\text { tienen que ver } \\
\text { tangencialmente } \\
\text { con la realidad } \\
\text { analizada y la } \\
\text { información } \\
\text { manejada en el } \\
\text { trabajo, además } \\
\text { no son } \\
\text { significativos para } \\
\text { la globalidad del } \\
\text { TFG. }\end{array}$ & $\begin{array}{l}\text { Las conclusiones } \\
\text { parten de la } \\
\text { realidad analizada } \\
\text { y de la } \\
\text { información } \\
\text { manejada en el } \\
\text { trabajo, sin } \\
\text { embargo son } \\
\text { parciales, están } \\
\text { incompletas o } \\
\text { están poco } \\
\text { definidas. }\end{array}$ & $\begin{array}{l}\text { Las conclusiones } \\
\text { son adecuadas a la } \\
\text { información que se } \\
\text { maneja y a la } \\
\text { realidad analizada, } \\
\text { son significativos } \\
\text { para la globalidad } \\
\text { del TFG. }\end{array}$ & $\begin{array}{l}15 \\
\%\end{array}$ \\
\hline
\end{tabular}




\begin{tabular}{|c|c|c|c|c|c|}
\hline Resultados & $\begin{array}{l}\text { Los resultados } \\
\text { reflejan } \\
\text { pobremente la } \\
\text { idea, careciendo de } \\
\text { la calidad mínima } \\
\text { deseable para ese } \\
\text { tipo de producto. }\end{array}$ & $\begin{array}{l}\text { Los resultados } \\
\text { reflejan } \\
\text { aceptablemente } \\
\text { la idea, pero } \\
\text { varios detalles- } \\
\text { algunos relevantes } \\
\text { - podrían haber } \\
\text { sido refinados } \\
\text { para una correcta } \\
\text { resolución. }\end{array}$ & $\begin{array}{l}\text { Los resultados son } \\
\text { idóneos y reflejan } \\
\text { adecuadamente } \\
\text { la propuesta, } \\
\text { pero algún detalle } \\
\text { - no relevante - } \\
\text { podría haber sido } \\
\text { refinado para una } \\
\text { mejor resolución.. }\end{array}$ & $\begin{array}{l}\text { Los resultados son } \\
\text { inteligentes } \\
\text { reflejando } \\
\text { excelentemente la } \\
\text { idea, además la } \\
\text { calidad es } \\
\text { sobresaliente para } \\
\text { ese tipo de } \\
\text { producto. }\end{array}$ & $\begin{array}{l}15 \\
\%\end{array}$ \\
\hline $\begin{array}{l}\text { Originalidad, } \\
\text { innovación }\end{array}$ & $\begin{array}{l}\text { El alumno solo } \\
\text { aporta síntesis de } \\
\text { informaciones } \\
\text { diversas. Realiza } \\
\text { una actuación } \\
\text { imitativa. No hace } \\
\text { ninguna aportación } \\
\text { novedosa. }\end{array}$ & $\begin{array}{l}\text { La propuesta } \\
\text { aporta soluciones } \\
\text { poco } \\
\text { innovadoras lo } \\
\text { que supone una } \\
\text { baja o escasa } \\
\text { incidencia en el } \\
\text { campo de } \\
\text { conocimiento al } \\
\text { que pertenece el } \\
\text { TFG. }\end{array}$ & $\begin{array}{l}\text { El alumno } \\
\text { plantea ciertas } \\
\text { ideas originales. } \\
\text { El trabajo } \\
\text { presenta todavía } \\
\text { algunas } \\
\text { aportaciones } \\
\text { débiles al campo } \\
\text { de conocimiento } \\
\text { al que pertenece } \\
\text { el TFG. }\end{array}$ & $\begin{array}{l}\text { El alumno plantea } \\
\text { ideas originales } \\
\text { suficientemente } \\
\text { fundamentadas y } \\
\text { argumentadas } \\
\text { desde la diferencia, } \\
\text { mejora o avance } \\
\text { con respecto al } \\
\text { entorno de } \\
\text { referencia } \\
\text { inmediato. El } \\
\text { trabajo representa } \\
\text { una aportación } \\
\text { importante al } \\
\text { campo de } \\
\text { conocimiento al } \\
\text { que pertenece el } \\
\text { TFG. }\end{array}$ & $\begin{array}{l}15 \\
\%\end{array}$ \\
\hline $\begin{array}{l}\text { Calidad de } \\
\text { los } \\
\text { contenidos }\end{array}$ & $\begin{array}{l}\text { Solo se contempla } \\
\text { una dimensión o } \\
\text { aspecto del tema } \\
\text { estudiado, Presenta } \\
\text { un nivel muy bajo } \\
\text { en cuanto a la } \\
\text { profundidad del } \\
\text { contenido. }\end{array}$ & $\begin{array}{l}\text { El tema del } \\
\text { trabajo se describe } \\
\text { de forma } \\
\text { superficial, no se } \\
\text { contemplan las } \\
\text { dimensiones } \\
\text { fundamentales ni } \\
\text { los aspectos del } \\
\text { tema necesarios. } \\
\text { Los contenidos se } \\
\text { tratan, en algún } \\
\text { caso, sin la } \\
\text { calidad adecuada } \\
\text { en un trabajo de } \\
\text { estas } \\
\text { características. }\end{array}$ & $\begin{array}{l}\text { Los contenidos } \\
\text { presentan un nivel } \\
\text { de profundidad } \\
\text { medio, adecuado } \\
\text { a los requisitos } \\
\text { del TFG. El tema } \\
\text { se trata teniendo } \\
\text { en cuenta las } \\
\text { dimensiones } \\
\text { adecuadas de } \\
\text { manera que, se } \\
\text { describe de forma } \\
\text { correcta y } \\
\text { completa a este } \\
\text { nivel. Las ideas } \\
\text { aportadas son de } \\
\text { calidad. }\end{array}$ & $\begin{array}{l}\text { El TFG incluye } \\
\text { contenidos que se } \\
\text { analizan en } \\
\text { profundidad. Se } \\
\text { consideran las } \\
\text { dimensiones } \\
\text { fundamentales y, } \\
\text { de modo completo, } \\
\text { todos los aspectos } \\
\text { clave del tema de } \\
\text { estudio. El } \\
\text { desarrollo de los } \\
\text { contenidos tiene un } \\
\text { elevado nivel de } \\
\text { calidad. }\end{array}$ & $\begin{array}{l}20 \\
\%\end{array}$ \\
\hline $\begin{array}{l}\text { Documentaci } \\
\text { ón gráfica }\end{array}$ & $\begin{array}{l}\text { Falta } \\
\text { documentación } \\
\text { gráfica básica para } \\
\text { la definición formal } \\
\text { del tema }\end{array}$ & $\begin{array}{l}\text { La documentación } \\
\text { gráfica presenta } \\
\text { errores } \\
\text { relevantes o muy } \\
\text { frecuentes }\end{array}$ & $\begin{array}{l}\text { Complementa la } \\
\text { documentación } \\
\text { escrita y aporta } \\
\text { los documentos } \\
\text { necesarios para la } \\
\text { definición formal } \\
\text { del tema, pero } \\
\text { parte de esa } \\
\text { documentación } \\
\text { gráfica presenta } \\
\text { algún error de } \\
\text { carácter menor. }\end{array}$ & $\begin{array}{l}\text { Complementa } \\
\text { perfectamente la } \\
\text { documentación } \\
\text { escrita, aportando } \\
\text { los documentos } \\
\text { necesarios para la } \\
\text { definición formal } \\
\text { del tema. Toda la } \\
\text { documentación } \\
\text { gráfica es correcta }\end{array}$ & $\begin{array}{l}15 \\
\%\end{array}$ \\
\hline
\end{tabular}


Estrategias para establecer un sistema que determine el seguimiento, estructura y evaluación de los trabajos final de grado en el Grado de Ingeniería en Diseño Industrial y Desarrollo de Productos

El último aspecto a evaluar es la exposición y defensas del trabajo. Esta evaluación la realiza el tribunal una vez acabada la defensa mediante otra la rúbrica en donde se evalúa la presentación y defensa del trabajo. La calificación será la media aritmética de las calificaciones de cada miembro del tribunal y tendrá un peso del $30 \%$ sobre la nota final.

\section{Resultados}

Los resultados esperados son documentos que permitan informar a profesores y alumnos sobre cómo proceder en el seguimiento, estructura y evaluación de los TFG.

Actualmente se dispone de un borrador del documento que se ha ofrecido a los profesores y alumnos de cuarto curso del Grado solicitándoles cualquier aportación que pueda mejorar la propuesta, estando a la espera de las mismas.

A modo de ejemplo se ofrece parte de los resultados obtenidos en cuanto a contenido (Tabla. 1 y 2) y rúbrica (Tabla.3) expuestos en los apartados anteriores. Se prentede que estos resultados sirvan de apoyo a los profesores que dirigen TFG en el Grado en Diseño Industrial y desarrollo de productos.

También se pretende ofrecer un documento guía para los miembros de tribunal, de modo que la evaluación del alumno se realice bajo los mismos criterios con idependencia de los miembros del tribunal asignados. Con todo ello se pretende -en la medida de lo posibleobjetivar el proceso de evaluación.

\section{Conclusiones}

Después de realizar el trabajo de estrategias para establecer un sistema que determine el seguimiento, estructura y evaluación de los trabajos final de grado en el Grado de Ingeniería en Diseño Industrial y desarrollo de productos se pretende implantar el nuevo método de evalución del TFG en el curso 2017-2018, pero no obstante, para este presente curso se les ha ofrecido a los profesores los esquemas de los contenidos del TFG mencionados anteriormente para que sus alumnos tutorizados puedan tener un guion fácil de seguir.

Para el próximo curso se está formando el grupo de profesores que realizará la tutorización de los TFG. Se incluyen profesores de distintas áreas de conocimiento, de manera que se tenga un seguimiento de los distintos partes o contenidos del TFG.

Al finalizar cada curso se realizará una exposición de todos los proyectos tutorizados, con la misma temática.

Con todo ello se ha perseguido unificar criterios dentro del trabajo fin de grado y que faciliten la labor de cada uno de los actores que intervienen en el proceso, alumno, director y miembros del tribunal. 
E. Juliá Sanchis, M. Valor Valor, L. Pla Ferrando, S. Sempere Ripoll, J.F. Picó Silvestre, M. BonetAracil, J.A. Martínez Cerver, A. Jordá Vilaplana

\section{Referencias}

AENOR (2009) Criterios generales para la elaboración formal de los documentos que constituyen un proyecto técnico. UNE 157001:2014 Madrid. AENOR

Bonilla Priego M.J.; Fuentes Moraleda, L.; Vacas Guerrero, C.; Vacas Guerrero, T. (2012) "Análisis del proceso de evaluación del Trabajo Fin de Grado en las nuevas titulaciones". Revista de Educación en Contabilidad, Finanzas y Administración de Empresas (2012, nº 3, p. 5-21).

Capó, A.J.; Guerrero, C.; Miró, J; Egea, A. (2012) “Elaboración de una rúbrica para la evaluación TFG y TFM de informática en la Universitat de les Illes Balears". En Actas Simposio-Taller JENUI 2012. Ciudad Real (2012, p. 17-24).

CIESE, (2016) Centro Universitario CIESE de la Fundación Comillas, guía de trabajo para profesores y alumnos, así como modelos de evaluación y rubricas de evaluación http://www.fundacioncomillas.es/centro-universitario/estudios-universitarios/grado/plan-deestudios/trabajo-fin-de-grado

EBRON (2014) Aplicación informática donde se gestionan los TFG en la UPV, Universitat Politécnica de Valencia. https://aplicat.upv.es/tfgtfmapp/seleccionRol.xhtml

Rekalde Rodríguez, I. (2011) “¿Cómo afrontar el trabajo fin de grado? Un problema o una oportunidad para culminar con el desarrollo de las competencias". Revista Complutense de Educación, (2011, vol. $22, n^{\circ} 2$, p. 179-193).

ROISS, S. (2015) "El Trabajo de Fin de Grado en el Grado de Traducción e Interpretación: evaluación de competencias y contenidos asociados al título. Estudio analítico y consecuencias didácticas". En Quaderns: revista de traducció ( 2015, $\mathrm{n}^{\circ}$ 22, p. 273-288)

Universidad de Granada, (2015) Plantillas para la evaluación de competencias en los TFG. http://docencia.ugr.es/pages/trabajo-fin-de-grado/instrumentos-evaluacion/rubricas

UPC, (2013) Universidad Politécnica de Cataluña y Universidad de Barcelona, marco normativo para realizar el TFG en el grado de estadística.

http://www.ub.edu/economiaempresa/grau/est/estadistica normativa TFG.pdf

(UPC, (2014) Universidad politécnica de Cataluña, Facultad de Náutica. Pasos, normativa, guía y plantilla de los TFG

http://www.fnb.upc.edu/content/treballs-fi-de-grau-i-m\%C3\%A0ster

UPV (2013) Normativa marco de trabajos fin de grado y fin de máster universitat politècnica de valència Aprobada en Consejo de Gobierno 7 de marzo de 2013

Valderrama, E.; Rullán, M.; Sánchez, F.; Pons, J.; Cores, F.; Bisbal, J. (2009) "La evaluación de competencias en los Trabajos Fin de Estudios". En Actas XV Jornadas de Enseñanza Universitaria de la Informática. Barcelona: (2009, p.405-412) 